\title{
Effects of surface modifications on deuterium retention in F82H and EUROFER exposed to low-energy deuterium plasmas
}

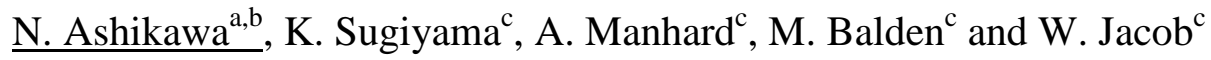 \\ ${ }^{a}$ National Institute for Fusion Science,Gifu, Japan \\ ${ }^{b}$ The Graduate University of Advanced Studies, Gifu, Japan \\ ${ }^{c}$ Max-Planck-Institut für Plasmaphysik, Garching, Germany,
}

Deuterium retention in two reduced-activation ferritic/martensitic (RAFM) steels, F82H (8Cr-2W) and EUROFER (9Cr$1 \mathrm{~W}$ ), was measured by nuclear reaction analysis and thermal desorption spectrometry after exposure to low-energy deuterium plasmas. In the RAFM steels, the deuterium retention was $10^{17}-10^{19} \mathrm{D} / \mathrm{m}^{2}$ and the major fraction of deuterium was retained in the near-surface layer.

The surface concentrations of tungsten increased with the $\mathrm{D}$ ion fluence and energy due to the selective sputtering of iron and chromium. Scanning electron microscopy images clearly indicated that the surface morphology changed with increasing $\mathrm{D}$ fluence (i.e. the grain boundaries became more visible because of erosion by ion bombardment). At a low fluence of about $10^{23} \mathrm{D} / \mathrm{m}^{2}$, deuterium retention was affected by surface modification in RAFMs.

Keywords: Deuterium Retention, F82H, EUROFER, First-wall, NRA, RBS

\section{Introduction}

Reduced-activation ferritic/martensitic (RAFM) steels such as F82H [1] and EUROFER [2] are candidate materials for fusion DEMO reactors [3-4]. For the design of DEMO reactors, hydrogen isotope retention is one of the most important issues related to tritium inventories, fuel recycling and fuel dilutions of tritium blanket modules, including plasma-facing walls. To understand the bulk fuelling retentions and tritium inventories of plasma-facing materials in DEMO reactors, analysing samples of RAFM materials that will be exposed to deuterium plasmas is necessary; in particular, the temperature of the first wall is expected to exceed $800 \mathrm{~K}$ and is related to the tritium-breeding efficiency in DEMO. However, experimental studies of the plasmawall interactions in the high-temperature region are not sufficient for RAFMs.

In this study, two RAFM steels, F82H (8Cr-2W) [1] and EUROFER (9Cr-1W) [2], were bombarded with steady-state deuterium plasmas under conditions comparable to the first-wall environment using the Pla-Q facility [5]. The effects of surface modifications on deuterium retention are discussed.

\section{Experimental setup and procedures \\ 2.1 Sample materials}

Two different sample samples, low-activity ferritic steel alloys F82H (8Cr-2W) and EUROFER (9Cr-1W), were used in this study. F82H was developed by JAEA [5-6] and was produced for the first wall of the vacuum vessel in JFT-2M. The treatment temperature was $630 \mathrm{~K}$ before installation; the baking temperature was $400 \mathrm{~K}$ during the experimental campaign at JFT-2M [7]. The atomic concentrations of main alloying elements to the Fe of the F82H sample material were $8 \% \mathrm{Cr}, 2 \% \mathrm{~W}$, $0.2 \% \mathrm{~V}$ and $0.06 \% \mathrm{Ta}$. Two different sample sizes were used: $10 \mathrm{~mm} \times 10 \mathrm{~mm} \times 1 \mathrm{~mm}$ and $15 \mathrm{~mm} \times 15 \mathrm{~mm} \times 1$ $\mathrm{mm}$. The sample surfaces exposed to the plasma were mechanically polished to a mirror finish by a Japanese company, and the surface roughness was less than 0.2 microns after the polishing treatment.

The atomic composition of the EUROFER was 9\% Cr, $1 \% \mathrm{~W}, 0.2 \% \mathrm{~V}, 0.05 \% \mathrm{Ta}, 0.4 \% \mathrm{Mn}$ in addition to Fe [2]. The sample size was $12 \mathrm{~mm} \times 15 \mathrm{~mm} \times 0.6 \mathrm{~mm}$. The EUROFER sample surfaces exposed to the plasma were also mechanically polished to a mirror finish at IPP, Germany.

After cleaning with alcohol, all samples were installed in a vacuum chamber for baking treatment. The samples were baked at $830 \mathrm{~K}$ for $2 \mathrm{~h}$.

\subsection{Experimental setup}

The Pla-Q facility employs a maximum input power of $1.2 \mathrm{~kW}$ and a $2.45-\mathrm{GHz}$ ECR plasma source that can generate steady-state plasmas. Plasmas travel downward and interact with the samples installed below the plasma source. The voltage of the sample holder is negatively biased. Typical achievable deuteron fluxes are on the order of $10^{19}$ to $10^{20} \mathrm{Dm}^{2} \mathrm{~s}^{-1}$ and are predominantly carried by $\mathrm{D}^{+3}$ ions [5]. Radiative heating caused by the plasma and heater are balanced during plasma exposure.

Hence, the temperatures of the samples facing the plasma were measured using an infrared camera, and the heater current was manually set. A hole was made on the sample holder for the temperature measurement, and the inside of this hole was blackened to achieve high emissivity. The temperatures measured using the infrared camera were calibrated using a thermocouple.

The flux was measured using a movable Langmuir probe. The diameter of the plasma column was approximately $50 \mathrm{~mm}$.

\subsection{Plasma experimental procedure}

After the baking procedure, the samples were exposed to plasmas in the Pla-Q facility. In this study, 
the primary working gas used for the plasma was deuterium, and sample bias voltages ranging from -75 to $-200 \mathrm{~V}$ were used. The temperature on the lower side of the sample was controlled by a heater, and silicon oils were used to maintain a temperature range of 350 to 450 K. For higher temperatures ranging from 550 to $780 \mathrm{~K}$, another sample holder with a heater was used. After plasma exposure, the samples were transported through air from the Pla-Q facility to a separate system [e.g. ion beam analysis (IBA) or thermal desorption spectrometry (TDS)]. Surface analyses were completed at least one day after plasma exposure; thus, the dynamic retention effects just after plasma exposure were negligible.

\subsection{Surface analysis}

The quantitative $\mathrm{D}$ depth profile was analysed using the $\mathrm{D}\left({ }^{3} \mathrm{He}, \mathrm{p}\right){ }^{4} \mathrm{He}$ reaction [6] in an IBA. For the RAFM specimens measured by NRA, the incident energy of the ${ }^{3} \mathrm{He}^{+}$beam was chosen as $690 \mathrm{keV}$. The $\mathrm{D}$ concentration within the near-surface layer (at depths up to about 0.5 $\mu \mathrm{m})$ was measured using the $\mathrm{D}\left({ }^{3} \mathrm{He}, \alpha\right) \mathrm{H}$ reaction at a ${ }^{3} \mathrm{He}^{+}$energy of $690 \mathrm{keV}$. The $\alpha$ particles were energyanalysed with a small-angle surface barrier detector at the laboratory scattering angle of $102^{\circ}$ and a solid angle of 7.65 msr. The $\alpha$ spectrum was transformed into a D depth profile using the SIMNRA program [8]. The protons from the $\mathrm{D}\left({ }^{3} \mathrm{He}, \mathrm{p}\right){ }^{4} \mathrm{He}$ nuclear reaction were counted using a wide-angle detector placed at a scattered angle of $135^{\circ}$ and a solid angle of $75.8 \mathrm{msr}$. As for the $\alpha$ spectrum analysis, the obtained proton spectrum was transformed into a D depth profile using the SIMNRA program. Deuterium retention analyses were performed using TDS (namely, TESS at IPP) to obtain the deuterium retained in the complete sample. The samples were heated from room temperature to $873 \mathrm{~K}$ at a rate of $0.25 \mathrm{~K} / \mathrm{s}$. The gases desorbed from the heated samples were measured using quadrupole mass spectrometry (QMS). Rutherford backscattering spectroscopy (RBS) was available in the IBA facility, and the compositions near the top surfaces of the specimens were analysed using a 1- $\mathrm{MeV}^{4} \mathrm{He}^{+}$beam with a beam incident angle of $75^{\circ}$ and a detector angle of $165^{\circ}$ to the surface normal.

The atomic concentrations on the sample surfaces were measured using X-ray photoelectron spectroscopy (XPS). A 400-W Mg X-ray source was used with an $800-\mu \mathrm{m}$ spot size. The following pass energies were used to achieve narrow profiles: $0.05 \mathrm{eV}$ for $\mathrm{Fe}, \mathrm{Cr}, \mathrm{O}$ and $\mathrm{C}$ and $0.1 \mathrm{eV}$ for $\mathrm{W}$. An argon ion gun was used to etch the samples and analyse their depth profiles. The erosion depths were measured with a contact-probe profilometer and used to calculate the sputtering rate.

The surface morphologies of the samples were observed using scanning electron microscopy (SEM; FEI, Helios Nanolab) at IPP, Garching.

\section{Results and discussion}

\subsection{Surface modifications and retentions in RAFMs}

Figure 1 shows the surface morphologies measured by SEM. These images were obtained on the surfaces of F82H and EUROFER after plasma bombardment and at different fluencies. The sample temperatures during plasma exposures were $450 \mathrm{~K}$ with a voltage bias of $-100 \mathrm{~V}$. The SEM image of F82H at a fluence of $1 \times$ $10^{23} \mathrm{D} / \mathrm{m}^{2}$ shows some lines with widths of a few tens of nanometres on the surface, which were considered to be the result of imperfect polishing. These small damages became less significant with increasing fluence. At a fluence of $1 \times 10^{25} \mathrm{D} / \mathrm{m}^{2}$, the surface morphologies observed in the images of both F82H and EUROFER are changed clearly with increasing deuterium fluence (i.e., the grain boundaries became more clearly visible because of erosion caused by ion bombardment).

Figure 2 shows the RBS spectra obtained in $\mathrm{F} 82 \mathrm{H}$ alloys after bombardment with deuterium plasma. Figure 2(a) shows the fluence dependence of tungsten concentration at a bias voltage of $-100 \mathrm{~V}$ and a sample temperature of $450 \mathrm{~K}$. The RBS counts at different fluences ranging from $1 \times 10^{23}$ to $5 \times 10^{23} \mathrm{D} / \mathrm{m}^{2}$ generate a smooth backscattering energy profile at around 920 $\mathrm{keV}$, indicating the presence of tungsten. At fluences exceeding $1 \times 10^{24} \mathrm{D} / \mathrm{m}^{2}$, the RBS counts around 920 $\mathrm{keV}$ increased, particularly for a fluence of $1 \times 10^{25}$ $\mathrm{D} / \mathrm{m}^{2}$. These higher RBS counts around a backscattering energy of $920 \mathrm{keV}$ are attributed to tungsten surface enrichment [6]. Hence, tungsten concentration is considered to increase with fluence and ion energy due to the selective sputtering of iron and chromium. Note that tantalum could also be enriched at the surface and contribute to these RBS counts; however, it was neglected because its concentration was 20 times lower in the material than that of tungsten.

Figure 2(b) shows the energy dependences of tungsten concentrations in F82H specimens. The RBS spectra exhibit an increase in tungsten with bias voltage above $-100 \mathrm{~V}$, as indicated by the increased counts at a backscattering energy of around $920 \mathrm{keV}$ [6]. Hence, tungsten concentrations are considered to increase with fluence and ion energy due to selective iron and chromium sputtering.

Figure 3 shows deuterium retention in F82H and EUROFER specimens after bombardment with deuterium plasma, as measured by NRA using a ${ }^{3} \mathrm{He}^{+}$ beam at $690 \mathrm{keV}$. The sample temperature was $450 \mathrm{~K}$, and two bias voltages were used: -75 and $-100 \mathrm{~V}$. These low bias voltages were chosen to reduce ion bombardment. In general, hydrogen isotope retention in metals is correlated with fluence. However, in this study,
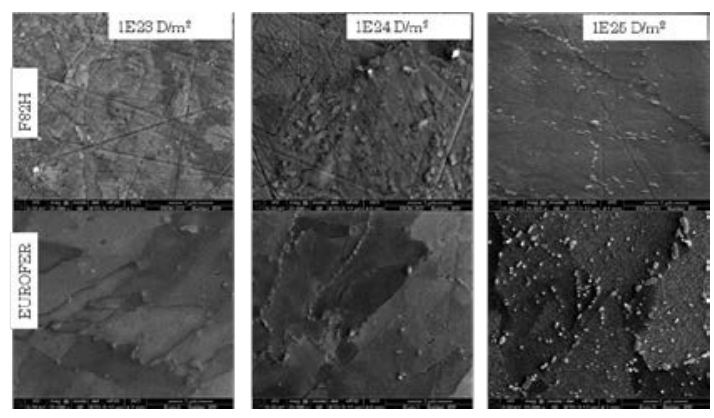

Fig. 1. SEM images obtained from the surface of F82H and EUROFER after deuterium bombardment at different fluencies. 
a higher retention was observed at low fluence, both in F82H and EUROFER. Based on the observations of surface modification (Fig. 1), we consider two reasons for the difference in fluence dependence. One is surface modification, which is related to erosion by ion bombardment and changing morphologies. The second reason is the contamination of the surfaces with impurities.

Trapped hydrogen isotopes such as tritium on grain boundaries have been reported on tungsten and steel specimens with ferrite-pearlite organization; tritium was charged by cathode electrolysis to form a ferrite-pearlite organization [9] and by a mixture of D-T gas at a
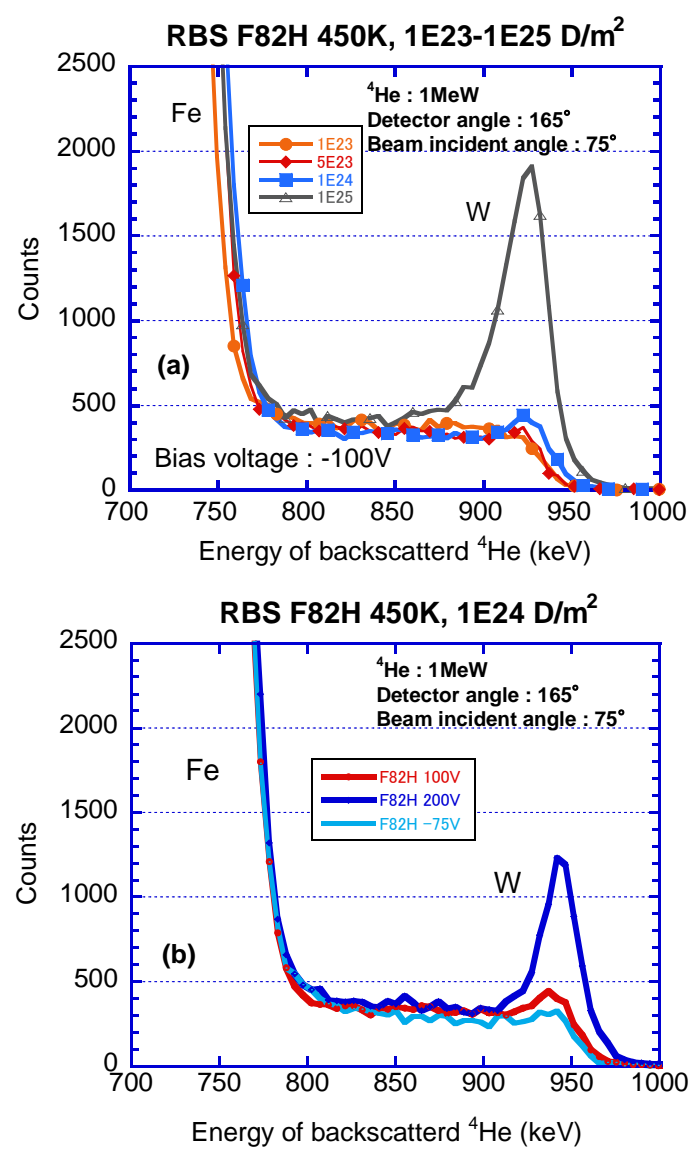

Fig. 2. RBS spectra obtained on F82H alloys after deuterium plasma bombardment with (a) different deuterium fluencies and (b) different bias voltages.

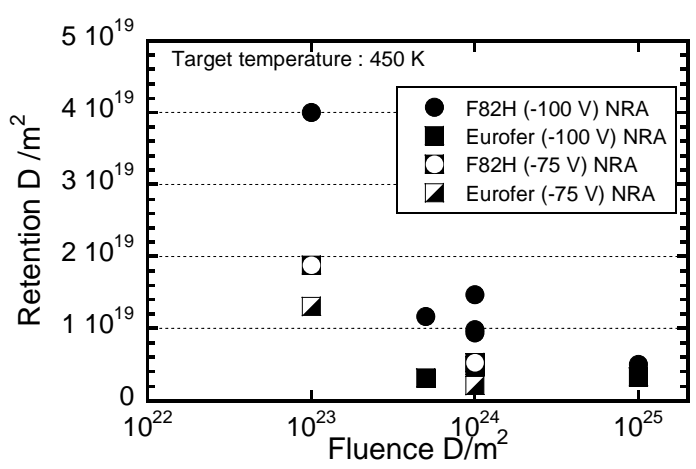

Fig. 3. Fluence dependence of deuterium retention after deuterium plasma bombardment. Retention is measured by NRA using ${ }^{3} \mathrm{He}^{+}$beam of $690 \mathrm{keV}$. pressure of $1 \mathrm{kPa}$ for over $5 \mathrm{~h}$ to form tungsten [10]. In both cases, the retained tritium was measured using tritium autoradiography. In this study, the surface morphologies at low fluences did not exhibit clear grains, and erosion had little influence (Fig. 1).

After cleaning with alcohol, the thickness of contaminants from air (e.g. carbon and oxygen impurities) on the polished surfaces of F82H specimens was only $2-3 \mathrm{~nm}$, as measured by sputtered XPS. This oxide layer is well known as the passive state film of steel. At a fluence of $1 \times 10^{24} \mathrm{D} / \mathrm{m}^{2}$, an eroded thickness of about $10 \mathrm{~nm}$ was observed when using deuterium plasmas. Below a fluence of $1 \times 10^{24} \mathrm{D} / \mathrm{m}^{2}$, the eroded thicknesses could not be evaluated using the weight loss measured by a microbalance. At low fluences on the order of $1 \times 10^{23} \mathrm{D} / \mathrm{m}^{2}$, thin layers of impurities remained, influencing the retention quantity.

The diffusion coefficient of hydrogen isotopes was calculated as [10]:

\section{$D=$ \\ $7.5 \times 10^{-4} \exp (-0.14[\mathrm{eV}] / \mathrm{kT})\left[\mathrm{cm}^{2} . \mathrm{s}^{-1}\right]$}

\section{(1)}

Using Eq. (1), the diffusion length, $\sqrt{D t}$, at $450 \mathrm{~K}$, was estimated to be $2.5 \mathrm{~mm}$ within $3000 \mathrm{~s}$ for a sample thickness of $1 \mathrm{~mm}$ in $\mathrm{F} 82 \mathrm{H}$. For short-term plasma operation, the hydrogen diffusion bulk profile does did not reach steady state; hence, deuterium retention was below the steady-state level. At a fluence of $1 \times 10^{24}$ $\mathrm{D} / \mathrm{m}^{2}$, the operation times were more than $3 \mathrm{~h}$, which is a result of the steady-state diffusion of deuterium in the specimen. Therefore, the retained deuterium was saturated.

\subsection{Temperature dependence of deuterium retention in RAFMs}

Figure 4 shows the deuterium retention in F82H and EUROFER after bombardment with deuterium plasma, as measured by NRA and TDS. Figure 4 depicts the protons measured by NRA using a $690-\mathrm{keV}^{3} \mathrm{He}^{+}$beam. The total deuterium fluence was about $1 \times 10^{24} \mathrm{D} / \mathrm{m}^{2}$ with an applied bias voltage of $-100 \mathrm{~V}$.

TDS analysis was completed only for the $\mathrm{F} 82 \mathrm{H}$

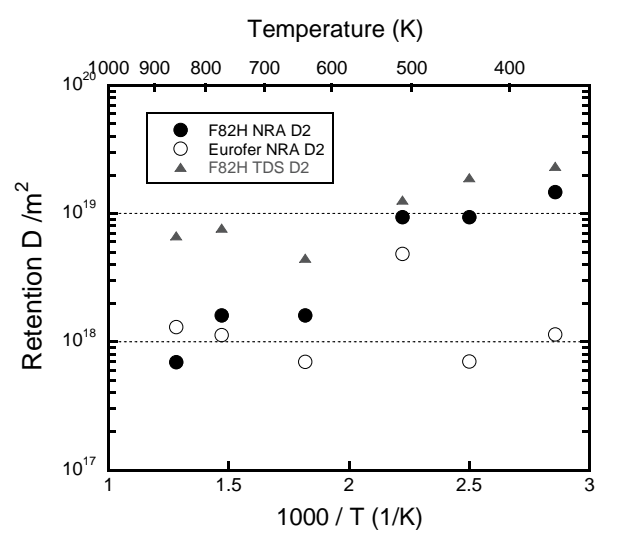

Fig. 4. Temperature dependence of deuterium retention on F82H and EUROFER. 
samples due to the limited number of specimens. The NRA and TDS retentions in F82H specimens at $350 \mathrm{~K}$ were different by an order of magnitude. However, these differences became smaller with increasing temperature. At $780 \mathrm{~K}$, the NRA and TDS retentions are different by only a factor of 2 . At higher temperatures (>650 K), the detected retention is small (near the detection limit of the analysers). For $\mathrm{F} 82 \mathrm{H}$, deuterium retention at lower temperatures was higher than that at higher temperatures for both the NRA and TDS measurements.

The $\mathrm{D}$ retention in EUROFER was measured only by NRA, and the measured retentions $\left(10^{17}-10^{18} \mathrm{D}\right.$ atoms $/ \mathrm{m}^{2}$; Fig. 4) were lower than those of $\mathrm{F} 82 \mathrm{H}$. The temperature dependence of EUROFER retention was not clear. The dependence of retention on fluence, as shown in Fig. 3, demonstrates the similar characteristics of retention in F82H and EUROFER. Regarding the temperature dependence of EUROFER retention, the retention quantity was affected by the surface conditions. The difference in retention between $\mathrm{F} 82 \mathrm{H}$ and EUROFER is shown in Fig. 4.

The thermal desorption spectra of F82H primarily exhibit a peak around $500 \mathrm{~K}$. In the temperature region of 350 to $450 \mathrm{~K}$, deuterium desorption decreased. The thermal desorption spectra over the temperature range of 550 to $780 \mathrm{~K}$ were similar to each other, and deuterium retention was of the order of $1 \times 10^{17} \mathrm{D} / \mathrm{m}^{2}$ (Fig. 4).

\section{Summary}

The deuterium retentions in F82H and EUROFER exposed to low-energy hydrogen plasma were investigated. In RAFMs, deuterium retention after bombardment with low-energy deuterium plasma was on the order of $10^{17}-10^{19} \mathrm{D} / \mathrm{m}^{2}$, and the major fraction of deuterium was retained in the near-surface layer.

The deuterium retention of the specimens after bombardment with deuterium plasma exhibited a temperature dependence in F82H. Deuterium retention was greater at lower temperatures than at higher temperatures; this phenomenon was confirmed by two separate analytical methods, NRA and TDS.

Tungsten enrichment was observed under the experimental conditions of this study (fluence $=1 \times 10^{24}$ $\mathrm{D} / \mathrm{m}^{2}$ and bias voltage $\left.=-100 \mathrm{~V}\right)$. Tungsten concentration increased with fluence and ion energy due to selective sputtering of iron and chromium. Based on SEM images, the surface morphologies clearly changed with increasing $\mathrm{D}$ fluence; that is, the grain boundaries became more visible due to erosion by ion bombardment. The difference between F82H and EUROFER was almost negligible.

In this paper, retained deuterium was observed near the top surface of RAFM. From the viewpoint of wall conditioning under vacuum, this result is advantageous for controlling wall recycling.

The hydrogen retention and surface characteristics of steel samples with and without helium bombardment will be investigated in the future.

\section{Acknowledgments}

This work was supported by the SOKENDAI Young Researcher Overseas Visit Program, JSPS KAKENHI Grant Number 26289352H, the NIFS budget UFFF028, KEMF058 and the JSPS-NRF-NSFC A3 Foresight Program in the field of Plasma Physics (NSFC: No.11261140328). The authors would like to thank Dr. Thomas Schwarz-Selinger and technical staffs of ion beam analysis and TDS in IPP for machine operations. The authors would like to thank Prof. Y. Hirooka for helpful suggestions, and Prof. A. Sagara for information regarding the FFHR. The authors would also like to thank Mr. Y. Miyo, Dr. T. Nakano, Dr. M. Fukumoto, Dr. A. Sakasai and Dr. H. Kubo of JAEA for their assistance with material preparation.

\section{References}

[1] H.Tanigawa, et al., J. Nucl. Mater. 417 (2011) 9 .

[2] B.van der Schaaf, et al., Fusion Eng. and Design, 69 (2003) 197.

[3] A.Sagara et al., Fusion Eng. and Design, 83 (2008) 1690.

[4] K.Tobita, et al., Nucl. Fusion 49 (2009) 075029.

[5] A. Manhard et al., Plasma Sources Sci. Technol (2011) 20015010.

[6] K. Sugiyama, et al., J. Nucl. Mater., 111-112 (2015) 628.

[7] K.Tsuzuki, et al., Nucl.Fusion 46 (2006) 966.

[8] M.Mayer, SIMNRA User's Guide, Tech. Rep. IPP 9/113, Max-Planck-Institut für Plasmaphysik, Garching, 1997.

[9] S.Nagata et al., J. Alloys and Compounds 356-357 (2003) 326.

[10] H. Zhou, et al, ICFRM-16 (2013). 